\title{
HOPE AND LIFE ENGAGEMENT OF PATIENTS WITH CANCER AT THE ADVANCED STAGE
}

\author{
Y. Cıracı Yasar, N. Nural, S. Gülhan \\ Institue of Health Sciences, Internal Medicine Nursing, TRABZON, Turkey.
}

\section{Introduction:}

Cancer is a disease that causes both physical and mental problems. These problems adversely affect the patient's life expectancies and engagements.

\section{Objectives}

The purpose of this study is to evaluate the implications of hope and life engagement in advanced cancer patients and to examine the factors that affect them.

\section{Methods}

This study performed as a cross-sectional and descriptive study on 74 cancer patients with advanced stage who hospitalized in Trabzon Kanuni Training and Research Hospital. Interview performed with emotional support by holding patient's hands and lasted about 1 hour. In the questionnaire the sociodemographic and emotional status of the patients were evaluated. As scale; the "integrative hope scale" and "life engagement scale" that were validated in Turkish validity were used. Parametric tests "Anova and t test" were used for the analysis of sociodemographic data and variables that could affect hope and life engagement. The significance level was accepted as 0.05 .

\section{Results}

The mean hope scale score of patients was $104,5946 \pm 6,80233$ and the mean life engegament scale score was $23,91 \pm 4,92340$. The variables which may affect mean hope score were age, fear of death, diagnosis, place of residence, support status, having children and gender. The variables which may affect mean life engagement score were place of residence, educational status, having children, level of pain, diagnosis, support status, agony.

\section{Conclusions}

Although the patients were advanced cancer patients, the level of hope and life engagement was found to be high. The environment they live in, their educational status, agony they experience, diagnosis, fear of death, support status and gender are influential in their desire to stay alive.

\begin{tabular}{|c|c|c|c|c|c|c|c|c|}
\hline Variables & $\begin{array}{l}\text { trust / faith } \\
x \pm 50\end{array}$ & $p$ & $\begin{array}{c}\text { lack of } \\
\text { perspective } \\
x \pm 50\end{array}$ & $p$ & $\begin{array}{c}\text { Positive Future } \\
\text { Orientation } \\
x \pm 50\end{array}$ & $p$ & $\begin{array}{c}\text { social } \\
\text { relationship/ } \\
\text { indivudial value } \\
\mathrm{X} \pm 5 \mathrm{~S}\end{array}$ & $p$ \\
\hline \multicolumn{9}{|l|}{ Educational status } \\
\hline No graduation & $41,80 \pm 5,70$ & & $13,70 \pm 2,15$ & & $19,60 \div 2,25$ & & $16,20 \pm 3,27$ & 0,701 \\
\hline Primary school" & $41,95 \pm 2,65$ & 0,401 & $14,95 \pm 3,03$ & 0,118 & $19,19 \pm 5,91$ & 0,409 & $15,23 \pm 1,25$ & \\
\hline $\begin{array}{l}\text { Secondary and } \\
\text { higher education }\end{array}$ & $43,66 \pm 4,04$ & & $13,50 \pm 2,23$ & & $19,66 \pm 1,87$ & & $14,50 \pm 1,67$ & \\
\hline \multicolumn{9}{|l|}{ Living place } \\
\hline Village* & $39,4 \pm \pm 3,46$ & & $14,33 \pm 2,56$ & & $19,00 \pm 0,97$ & & $14,66 \pm 1,67$ & \\
\hline Town & $43,41+3,52$ & 0,03 & $13,83 \pm 2,94$ & 0,425 & $19,41 \pm 1,41$ & 0,465 & $15,41 \pm 1,28$ & 0,161 \\
\hline city & $42,81 \pm 4,23$ & & $14,81 \pm 2,71$ & & $19,56 \pm 1,86$ & & $15,81 \pm 2,59$ & \\
\hline \multicolumn{9}{|l|}{ Agony } \\
\hline Yes * & $41,76 \pm 4,66$ & & $13,70 \pm 3,01$ & & $19,35 \pm 1,55$ & & $15,11 \pm 2,69$ & \\
\hline No & $41,90 \pm 3,90$ & 0,403 & $15,54 \pm 2,23$ & 0,047 & $19,27 \pm 1,51$ & 0,243 & $15,63 \pm 1,25$ & 0,560 \\
\hline silent" & $43,33 \pm 3,06$ & & $14,22+2,41$ & & $19,55 \pm 1,61$ & & $15,66 \pm 1,60$ & \\
\hline \multicolumn{9}{|l|}{ Fear of death } \\
\hline Yes & $42,56 \pm 3,38$ & & $15,06 \pm 2,80$ & & $19,50 \pm 1,606$ & & $15,56 \pm 2,63$ & \\
\hline No: & $39,28 \pm 3,45$ & 0,01 & $14,71 \pm 1,69$ & 0,061 & $19,28 \pm 7,91$ & 0,243 & $15,00 \pm 1,10$ & 0,709 \\
\hline Silent: & $43,21 \pm 4,63$ & & $13,42+2,76$ & & $19,2 a+1,73$ & & $15,42 \pm 1,79$ & \\
\hline \multicolumn{9}{|l|}{ Despair } \\
\hline Yes & $41,00 \pm 3,59$ & 0,154 & $14,50 \pm 2,50$ & 0,902 & $18,03 \pm 1,37$ & 0,052 & $15,00 \pm 1,21$ & 0,392 \\
\hline No & $42,45 \pm 4,17$ & & $14,41+2,87$ & & $19,54 \pm 1,51$ & & $15,45 \pm 2,40$ & \\
\hline \multicolumn{9}{|l|}{ Reufulness } \\
\hline Yes & $42,77 \pm 4,22$ & 0,136 & $14,50 \pm 2,93$ & 0,638 & $19,72+1,40$ & 0,02 & $15,45 \pm 2,39$ & 0,795 \\
\hline No: & $41,33 \pm 3,000$ & & $14,20+2,49$ & & $18,06 \pm 1,61$ & & $15,33 \pm 1,60$ & \\
\hline \multicolumn{9}{|l|}{$\begin{array}{l}\text { Need of } \\
\text { psychological } \\
\text { support }\end{array}$} \\
\hline Yes & $40,35 \pm 4,22$ & 0,004 & $15,21+2,36$ & $0, \infty 01$ & $19,00 \div 1,44$ & 0,094 & $15,73 \pm 2,27$ & 0,060 \\
\hline No & $43,30+3,68$ & & $13,00+202$ & & $19,60 \pm 1,570$ & & $14,05 \pm 1,67$ & \\
\hline
\end{tabular}

\begin{tabular}{|c|c|c|c|c|c|}
\hline Variables & $\begin{array}{c}n \\
(\mathrm{~N}=74)\end{array}$ & $\begin{array}{l}\text { Integrative Hope scale } \\
\qquad \mathrm{X} \pm \mathrm{SD}\end{array}$ & $p$ & $\begin{array}{l}\text { Life Engagement } \\
\qquad x \pm 50\end{array}$ & $p$ \\
\hline \multicolumn{6}{|c|}{ 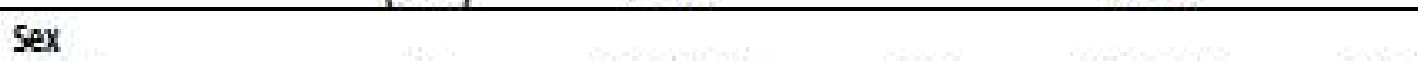 } \\
\hline Female & 50 & $103,24 \pm 5,17$ & \multirow[t]{2}{*}{0,261} & $24,24 \pm 4,27$ & \multirow[t]{2}{*}{0,490} \\
\hline Male & 24 & $106,16 \pm 9,27$ & & $23,25 \pm 6,10$ & \\
\hline \multicolumn{6}{|l|}{ Age } \\
\hline $18-44$ & 12 & $107,16 \pm 6,91$ & \multirow{3}{*}{0,277} & $26,00 \pm 3,95$ & \multirow{3}{*}{0,139} \\
\hline $45-64$ & 26 & $104,44 \pm 7,99$ & & $24,38 \pm 4,94$ & \\
\hline$>65$ & 36 & $103,55 t 5,60$ & & $22,80 \pm 5,04$ & \\
\hline \multicolumn{6}{|l|}{ Educational status } \\
\hline No graduation & 20 & $105,90 \pm 10,42$ & \multirow{3}{*}{0,268} & $23,90+5,41$ & \multirow[t]{3}{*}{0,033} \\
\hline Primary school" & 42 & $103,47 \pm 3,56$ & & $23,00 \pm 4,89$ & \\
\hline $\begin{array}{l}\text { Secondary and higher } \\
\text { education }\end{array}$ & 12 & $106,33 \pm 7,78$ & & $27,16 \pm 2,44$ & \\
\hline \multicolumn{6}{|l|}{ Marital status } \\
\hline Married & 52 & $103,53 \pm 4,90$ & \multirow[t]{2}{*}{0,113} & $23,34 \pm 4,85$ & \multirow[t]{2}{*}{0,070} \\
\hline Single/widow & 22 & $107,09 \pm 9,65$ & & $25,27 \pm 4,92$ & \\
\hline \multicolumn{6}{|l|}{ Having children } \\
\hline Wo children & 8 & $108,25 \pm 0,29$ & \multirow{3}{*}{0,156} & $25,75 \pm 4,92$ & \multirow{3}{*}{0,417} \\
\hline $1-2$ children & 8 & $101,75+3,95$ & & $22,50 \pm 5,47$ & \\
\hline$\$ 3$ children & 58 & $104,40 \pm 6,77$ & & $23,86 \pm 4,06$ & \\
\hline \multicolumn{6}{|l|}{ Living with } \\
\hline Lonely & 4 & $102,00 t 2,30$ & \multirow[t]{2}{*}{0,096} & $21,00+2,30$ & \multirow[t]{2}{*}{0,066} \\
\hline Fanily & 70 & $104,74 \pm 6,95$ & & $24,00 \pm 4,98$ & \\
\hline \multicolumn{6}{|l|}{ Living place } \\
\hline Village" & 18 & $100,77 \pm 4,54$ & \multirow{3}{*}{0,018} & $20,66 \pm 4,44$ & \multirow{3}{*}{0,003} \\
\hline Town & 24 & $106,41 \pm 5,11$ & & $25,58 \pm 4,92$ & \\
\hline city & 32 & $105,37 \pm d, 15$ & & $24,50 \pm 4,42$ & \\
\hline
\end{tabular}

\begin{tabular}{|c|c|c|c|c|c|}
\hline Variables & $\begin{array}{c}n \\
(N=74)\end{array}$ & $\begin{array}{l}\text { integrative hope scale } \\
x \neq S D\end{array}$ & $p$ & $\begin{array}{l}\text { Life Engagement } \\
X \pm \text { sD }\end{array}$ & $p$ \\
\hline \multicolumn{6}{|l|}{ Diagnosis } \\
\hline Gisca & 48 & $103,00 \pm 4,26$ & \multirow{3}{*}{0,302} & $22,87 \pm 5,12$ & \multirow{3}{*}{0,09} \\
\hline Lung ca & 16 & $108,00 \pm 10,27$ & & $25,62+4,37$ & \\
\hline other" & 10 & $106,80 \pm 8,03$ & & $26,20 \pm 3,29$ & \\
\hline \multicolumn{6}{|c|}{ Knowladge of disease } \\
\hline Enough & 36 & $104,72 \pm 5,80$ & \multirow{3}{*}{0,098} & $23,94 \pm 4,56$ & \multirow{3}{*}{0,81} \\
\hline Partially enough & 24 & $102,66 \pm 3,87$ & & $23,50 \pm 4,99$ & \\
\hline Not enough & 14 & $107,57 \pm 11,22$ & & $24,57 \pm 5,91$ & \\
\hline \multicolumn{6}{|l|}{ Pain intensity } \\
\hline Low & 20 & $105,50 \pm 5,79$ & \multirow{3}{*}{0,551} & $24,50 \pm 4,28$ & \multirow{3}{*}{0,54} \\
\hline intensive & 10 & $102,60 \pm 4,92$ & & $25,00 \pm 3,77$ & \\
\hline So intensive & 44 & $104,63 \pm 7,57$ & & $23,40 \pm 5,41$ & \\
\hline \multicolumn{6}{|l|}{ Agony } \\
\hline Yes & 34 & $104,52 \pm 8,21$ & \multirow{3}{*}{0,026} & $23,47 \pm 5,63$ & \multirow{3}{*}{0,25} \\
\hline No & 22 & $106,33 \pm 3,49$ & & $25,22 \pm 4,96$ & \\
\hline Silent" & 18 & $103,27 \pm 6,35$ & & $23,54 \pm 3,52$ & \\
\hline \multicolumn{6}{|c|}{ Fear of sleep / darkness } \\
\hline Yes & 44 & $104,54 \pm 7,36$ & \multirow[t]{2}{*}{0,938} & $24,09 \pm 5,40$ & \multirow[t]{2}{*}{0,706} \\
\hline No & 30 & $104,66 \pm 5,99$ & & $23,66 \pm 4,19$ & \\
\hline \multicolumn{6}{|l|}{ Fear of death } \\
\hline Yes & 32 & $104,56 \pm 7,20$ & \multirow{3}{*}{0,038} & $24,37 \pm 4,72$ & \multirow{3}{*}{0,309} \\
\hline No ". & 14 & $100,85 \pm 4,48$ & & $24,21+5,64$ & \\
\hline Silent & 28 & $106,50 \pm 6,69$ & & $22,20+3,62$ & \\
\hline \multicolumn{6}{|l|}{ Despair } \\
\hline Yes & 48 & $102,50 \pm 3,24$ & \multirow{3}{*}{0,048} & $23,25 \pm 4,45$ & \multirow{3}{*}{0,402} \\
\hline $\mathrm{No}^{*}$ & 24 & $105,04 \pm 7,44$ & & $24,25 \pm 5,25$ & \\
\hline Silent".* & 2 & $119,00 \pm 0,00$ & & $24,00+0,00$ & \\
\hline \multicolumn{6}{|l|}{ Reufulness } \\
\hline Yes & 44 & $105,45 \pm 7,61$ & \multirow[t]{2}{*}{0,161} & $24,90 \pm 4,87$ & \multirow[t]{2}{*}{0,034} \\
\hline No & 30 & $103,33 \pm 5,27$ & & $22,46 \pm 4,69$ & \\
\hline \multicolumn{6}{|l|}{ Longing } \\
\hline Yes & 38 & $104,42 \pm 7,98$ & \multirow[t]{2}{*}{0,822} & $23,73 \pm 5,62$ & \multirow[t]{2}{*}{0,744} \\
\hline No & 36 & $104,77 \pm 5,38$ & & $24,11 \pm 4,11$ & \\
\hline \multicolumn{6}{|c|}{ Need of psychological support } \\
\hline Yes & 46 & $105,43+7,11$ & 0,160 & $21,64 \pm 5,49$ & 0,004 \\
\hline No & 28 & $103,21 \pm 6,13$ & & $25,30 \pm 4,00$ & \\
\hline
\end{tabular}

